\title{
Dendrimers as novel class of polymeric corrosion inhibitors:
}

\section{A review}

\author{
C. Verma, ${ }^{1,2} *$ E.E. Ebenso ${ }^{1,2}$ and M.A. Quraishi ${ }^{3,4}$
}

${ }^{I}$ Department of Chemistry, School of Chemical and Physical Sciences, Faculty of Natural and Agricultural Sciences, North-West University, Private BagX2046, Mmabatho 2735, South Africa

${ }^{2}$ Material Science Innovation \&Modelling (MaSIM) Research Focus Area, Faculty of Natural and Agricultural Sciences, North-West University, Private Bag X2046, Mmabatho 2735, South Africa

${ }^{3}$ Department of Chemistry, Indian Institute of Technology, Banaras Hindu University, Varanasi 221005, India

${ }^{4}$ Center of Research Excellence in Corrosion, Research Institute, King Fahd University of Petroleum \& Minerals, Dhahran 31261, Saudi Arabia *E-mail: chandraverma.rs.apc@itbhu.ac.in; cbverma38@gmail.com

\begin{abstract}
Dendrimers are a new class of highly branched, nano-sized, monodisperse macromolecules that have several fascinating physical and chemical characteristics. They are highly soluble in the polar solvents, contain several surface functionality and high surface area which makes them suitable reagents for industrial and biological applications. Recently, several dendrimers have been investigated as effective corrosion and scale inhibitors for metallic and silica corrosion using experimental and theoretical methods. Generally, dendrimers become effective by forming insoluble metal-dendrimer or silica-dendrimer composite on the surface. Their inhibition performance of dendrimers have been measured using several experimental such as weight loss, electrochemical (EIS and PDP) and surface (EDX, SEM and AFM) as well as computation techniques such as molecular dynamics simulation and DFT based quantum chemical calculations. The interactions between metal surface and dendrimers can be explain through some common parameters such as $E_{\mathrm{HOMO}}, E_{\mathrm{LUMO}}, \Delta E$, electronegativity $(\chi)$, hardness $(\eta)$, softness $(\sigma)$, dipole moment $(\mu)$ and fraction of electron transfer $(\Delta N)$. Present book chapter deals with detail description on the corrosion inhibition and anti-scaling behaviours of dendrimers for metals and silica in several electrolytic media.
\end{abstract}

Keywords: dendrimers, corrosion inhibitors, mixed type, Langmuir adsorption isotherm, DFT study.

Received: August 1, 2018. Published: November 21, 2018

doi: $\underline{10.17675 / 2305-6894-2018-7-4-8}$ 


\section{Introduction}

\subsection{Corrosion and its adverse effect}

Most of the metals are highly unstable and readily react with the components of the surrounding environment by chemical or electrochemical mechanism and convert some more stable forms (rusts and scales). This is an irreversible, spontaneous and damaging phenomenon and commonly termed as corrosion. Corrosion causes huge losses of metallic materials and their properties which ultimately results into big economic and safety losses throughout the world. Therefore, corrosion has become worldwide problem for developed as well as developing nations to be considered by educational as well as industrial sectors $[1,2]$. A study conducted by National Association of Corrosion Engineers (NACE; 2002) revealed that in the year 1998, United State annually losses about US \$276 billion which equates about $3.1 \%$ of total U.S. gross domestic product (GDP) [3,4]. The cost of corrosion in U.S. exceeded more than US \$2.2 trillion in the year 2011. In India, according to $1^{\text {st }}$ Global Corrosion Summit held in New Delhi, corrosion causes loss of about Rs. 2 lakhs crores (US $\$ 45$ billion) in the year $2011[3,4]$. According to the most recently published data from the NACE study reveals that annually (worldwide) corrosion causes loss of around US \$2.5 trillion which equates about $3.4 \%$ of the world GDP [3,4]. The annual loss due to corrosion in India and South Africa are about Rs. 6 lacks crores (US \$100-billion) and R130-billion (i.e. about US \$ 9.6 billion), respectively [3, 4].

\subsection{Factors affecting corrosion}

Corrosion is a spontaneous process in which metals (except gold, silver and platinum) and alloys undergo rapid oxidation in the presence of constituents of the surrounding environment. Because corrosion is a spontaneous phenomenon its rate mainly depends upon the change in the standard Gibbs' free energy $\left(\Delta G_{\mathrm{corr}}^{0}\right)$. The greater change in standard Gibbs' free energy ( $\Delta G_{\text {corr }}^{0}$; in negative) results into higher chemical spontaneity or corrosion rate and vice versa [5-7]. Generally, rate of metallic corrosion influenced by several factors such as solution temperature, nature of metals and electrolytes and presence of impurities [7-11]. Besides these environmental factors, corrosion rate is also influenced by nature of rusts and scales accumulated on the metallic surface which may or may not be protective. The inhibitive and corrosive nature of the accumulated rusts and scales collected on the metallic surface can be determined by Pilling-Bedworth ratio which is generally expressed by $M d / m n D[12,13]$. In the Pilling-Bedworth ratio $M$ and $D$ are respectively, represent the molecular weight and density of the rusts and/or scales gathered on the surface. Whereas, $m$ and $d$, respectively represent the atomic weight and density of the metal under consideration and $n$ represents the metallic number in the rusts and scales (corrosion products); for example in $\mathrm{Fe}_{2} \mathrm{O}_{3}, \mathrm{Al}_{2} \mathrm{O}_{3}$ and $\mathrm{Fe}_{3} \mathrm{O}_{4}, n$ are 2, 2 and 3 respectively $[12,13]$. In general, for case $M d / m n D<1$, it is expected that volume of metal surface is larger than that of the corrosion products (rusts/scales) and therefore surface film is non- 
protective as it contains exposed metallic area. Conversely, for case $M d / m n D>1$, it is expected that volume of metal surface is smaller than corrosion products and in this situation surface film will be more compact, compressed and protective in nature.

\subsection{Corrosion monitoring techniques}

The corrosion is a dangerous phenomenon and therefore previously several methods of its protection have been developed by properly applying then the cost of corrosion can be reduced from 15\% (US \$ 375 billion) to $35 \%$ (US \$ 875 billion). The available methods of corrosion protection is shown in the Figure 1. Among the commonly used corrosion control methods such as coating, plating, potential change, design improvement and environmental modification, use of synthetic corrosion inhibitors is the most common and effective methods due to their ease synthesis and application as well as cost effective nature $[14,15]$. The anodic inhibitors are also known as passivation inhibitors as they generally inhibit corrosion by forming the passive film on the metal surface that causes a large shift in the corrosion potential $\left(E_{\text {corr }}\right)$ towards anodic direction [16]. There are several anodic inhibitors such as nitrate, chromate and nitrite, known those can passivate metallic surface even in the absence of oxygen and called as oxidizing anodic (anions) inhibitors. On the other hands there are several other inhibitors such as molybdate, tungstate and phosphate those can passivate metallic surface only in the presence of oxygen. These passivating inhibitors are known as non-oxidizing anions. The cathodic inhibitors either retards the cathodic reaction or precipitates on the cathodic area which retort the rate of diffusion of reducible species like $\mathrm{H}^{+}$and thereby increase the surface impedance $[16,17]$. The cathodic inhibitors generally act by three different mechanisms namely, cathodic precipitates, cathodic poisons and oxygen scavengers. The sodium sulphide and hydrazine react with the surrounding oxygen and behave as most common oxygen scavengers. Arsenic and antimony are two most common cathodic poisons as they retard the combination of hydrogen ions during their cathodic reduction. The calcium, magnesium and zinc easily form oxides which generally precipitate on the cathodic area and act as cathodic precipitates [18-20]. It is recalled that out of various corrosion control methods, the use of organic inhibitors is the most common and popular method.

These inhibitors contain several heteroatoms those exist in the form of polar functional groups like $-\mathrm{NO}_{2},-\mathrm{OH},-\mathrm{OCH}_{3},-\mathrm{NMe}_{2},-\mathrm{COOC}_{2} \mathrm{H}_{5},-\mathrm{CN}$ and $-\mathrm{O}-$ etc. [21-23]. The polar functional groups along with the non-bonding electrons of heteroatoms and pi-electrons of the multiple bonds behave as adsorption centres during interactions between inhibitor and metal surface. The interactions of inhibitors with metal result into their adsorption on the surface which is behaves as barrier for corrosion process [21-27]. 


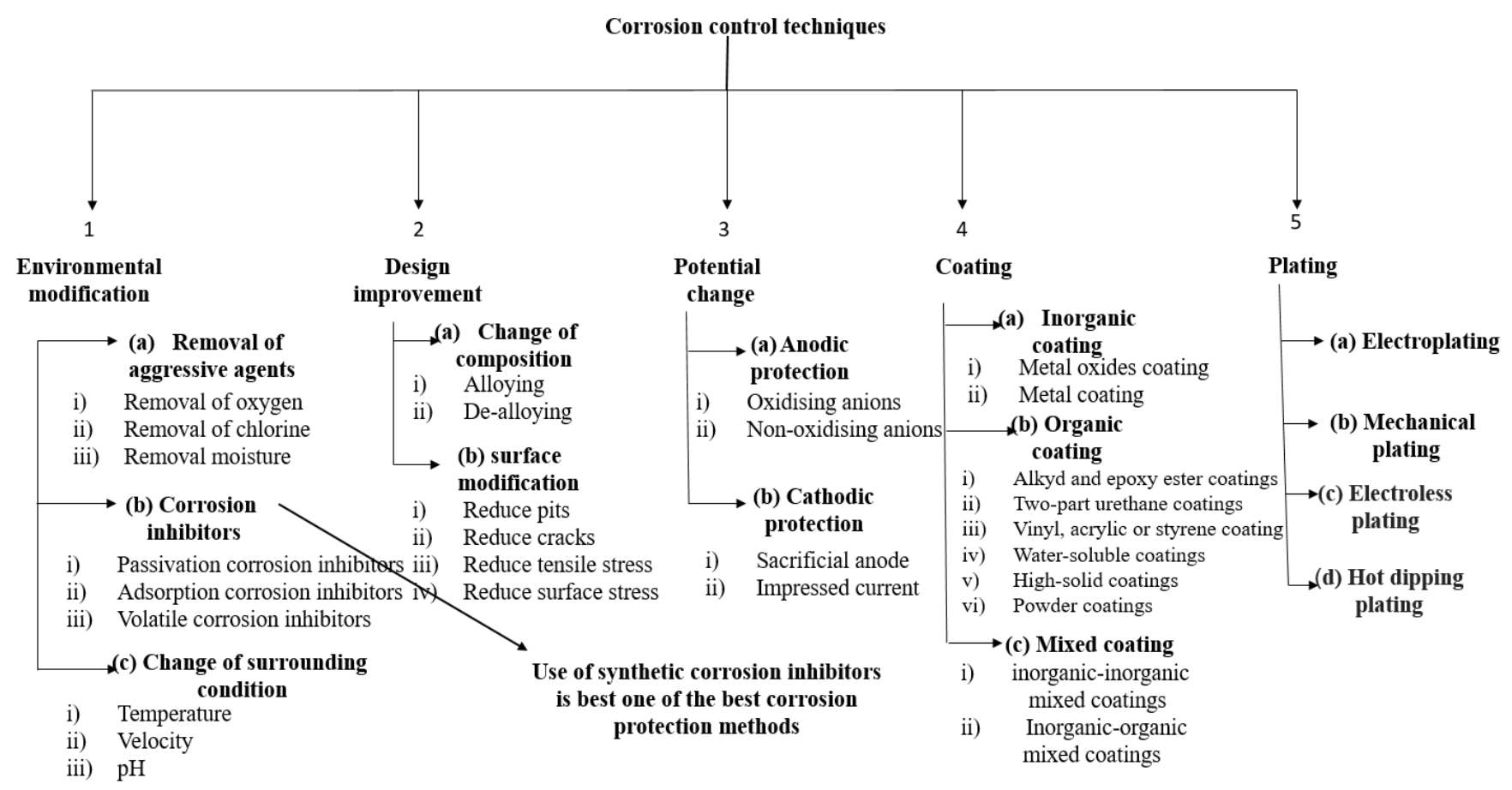

Figure 1. Methods of corrosion protection.

\subsection{Salient features and classification of dendrimers}

Dendrimers are highly branched polymeric materials which consist of three different architecture components namely, core, branches and terminal functional groups [28]. Core constitutes the central part of the dendrimeric architecture which me be either an atom or a molecule with at least two identical functionality (chemical functional groups). The branches are the repeat units of dendrimers which start from core i.e. focal point. The geometric repetition of the dendrimeric branches results into the formation of radially centric layers called as "generations" [28,29]. The terminal functional groups are positioned at the periphery of the dendrimeric architecture and is the most critical factor which determines the property of the dendrimers. The nano-sized dendrimeric polymers differ from the linear (ordinary) polymers in the sense that dendrimers are highly precise controlled architecture with high surface functionality. Moreover, in contrast to linear, dendrimeric polymers are synthesized by controlled chemical reaction that leads to the construction of globular, monodisperse and macromolecular polymeric architecture [28, 29]. The dendrimers architectures can be further classified into dendrigraft polymers, hyper-branched polymers, dendrons and dendrimers [28, 29]. There are two different approaches namely, divergent and convergent are being utilized for the synthesis of variety of dendrimeric polymers. In divergent approach, synthesis of the dendrimers starts from the core and rapidly grown away to the periphery. In contrast, convergent synthesis starts from the periphery and proceeds toward interior before their joining two pre-synthesized dendrons to the dendrimer core [28, 29]. Several types of dendrimers are being synthesized 
using various core and surface functional molecules. Figure 2 briefly describes the different types of dendrimers studied in the literature.

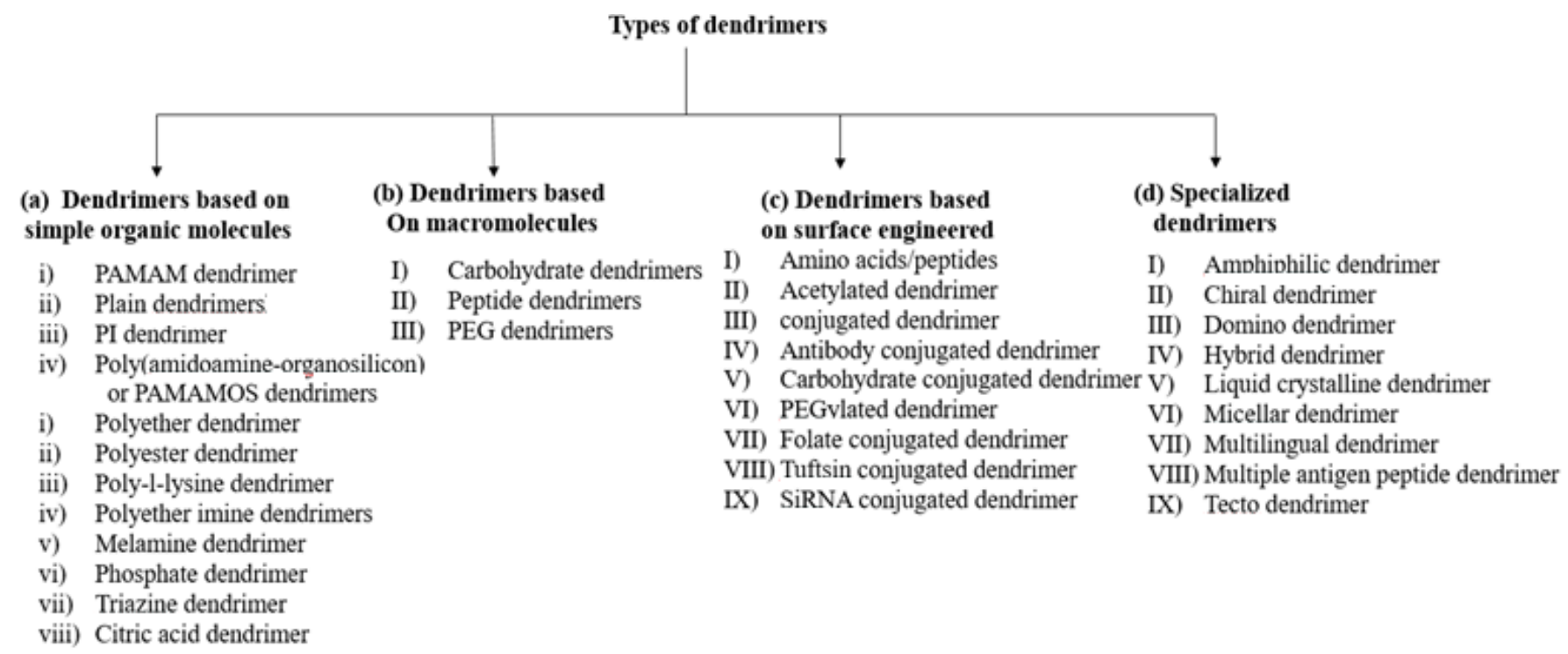

Figure 2. Types of dendrimers.

\section{Dendrimers as corrosion inhibitors}

Most of the known corrosion inhibitors are small organic molecules and therefore either they occupy lower metallic area or require high concentration as compared to the polymeric corrosion inhibitors [30-32]. Several kinds of the polymers have been used as corrosion inhibitors for metals and alloys in the different kinds of electrolytic media. Literature survey reveals that recently few dendrimeric polymers have also been investigated as effective corrosion inhibitors particularly for mild steel in acidic and $\mathrm{CO}_{2}$ solutions. Due to presence of long molecular chain, their ease and effective synthesis, nano-sized (high surface area), high solubility in the polar electrolytic media like $\mathrm{H}_{2} \mathrm{O}$ and $\mathrm{HCl}$ and presence of polar functional groups at the periphery, dendrimers can adsorb and act as good corrosion inhibitors for metallic corrosion in the different electrolytic media. Moreover, due to their polymeric nature they cover the larger surface area and form relatively more effective corrosion barrier on the metal surfaces which isolates them from surrounding environments and thereby inhibit metals corrosion. Khaled and co-workers [33] study the inhibition property of ethylenediamine cored, hydrophilic and highly branched PAMAM dendrimer on steel corrosion in $1 \mathrm{M}$ hydrochloric acid medium using potentiodynamic polarization (PDP), electrochemical impedance spectroscopy (EIS) and molecular dynamics simulation methods. These authors studied the inhibition effect of PAMAM at 5, 15, 20, 30, 50 and $100 \mathrm{ppm}\left(\mathrm{mg} \mathrm{L}^{-1}\right)$ concentrations and observed that it shows the maximum inhibition efficiency of $88.78 \%$ at $100 \mathrm{ppm}$ concentration. PDP study revealed that investigated PAMAM dendrimer behaves as cathodic type inhibitor and EIS study showed that addition of the PAMAM in the corrosive environment increases the polarization resistance and thereby retards the metallic corrosion. The molecular dynamics 
simulation study suggests that the PAMAN dendrimer inhibits steel corrosion by adsorbing on the surface in which nitrogen atoms as well as $\pi$-electrons act as adsorption centres.

The inhibition property of ethylenediamine core based three generation namely, DENG-G $\mathrm{G}_{0}, \mathrm{DENG}_{\mathrm{G}} \mathrm{G}_{1}$ and DENG- $\mathrm{G}_{2}$ dendrimers on mild steel corrosion in the solution of $1 \mathrm{M} \mathrm{HCl}$ using weight loss, PDP, EIS, scanning electron microscopy (SEM) and energy dispersive X-ray (EDX) techniques has been studied by Verma and Quraishi [34]. All the studied dendrimers act by adsorbing on the surface through their non-bonding electrons of oxygen and nitrogen as well as $\pi$-electrons of $>\mathrm{C}=\mathrm{O}$ bonds. Their adsorption on the test material obeyed the Langmuir adsorption isotherm. The studied dendrimers act as mixed type inhibitors as depicted by their polarization study. The inhibition efficiency of the tested dendrimers at their optimum concentration of $25 \mathrm{ppm} / 11.2 \times 10^{-5} \mathrm{M}$ follows the sequence: DENG-G ${ }_{0}(94.78 \%)<$ DENG-G $_{1}(96.08 \%)<$ DENG-G $_{2}(96.95 \%)$. The EIS results suggest that the dendrimers adsorb on the interfaces of metal and electrolyte and act as barrier for corrosive metallic dissolution. The adsorption mechanism of corrosion inhibition derived from weight loss, EIS and PDP methods was supported by SEM and EDX analyses. SEM images in presence of optimum concentration of the dendrimers show significant smoothness in the surface morphology as compared to the SEM image of uninhibited metal specimen. This finding revealed that dendrimers adsorb on the metal surface and protect from corrosion. The EDX spectra of inhibited metallic specimens showed signals for the presence of nitrogen and oxygen which was absent in the EDX spectrum of uninhibited mild steel specimen conforming that dendrimers adsorbed on the metal specimens. Moreover, the intensity of the signals correspond to nitrogen and oxygen enhance from going DENG- $G_{0}$ to $D E N D-G_{2}$ which suggests that effectiveness of the adsorption increases in the same order.

Zhang et al. [35] reported the inhibition properties of ethylenediamine based polyamidoamine (PAMAM) dendrimer in the combination with sodium silicate for carbon steel in soft water using weight loss, EIS, PDP, EDX and atomic force microscopic (AFM) methods. The study reveals that investigated dendrimer is an eco-friendly inhibitor and its lower doses are required for effective corrosion inhibition. Both the tested components i.e. PAMAM and sodium silicate inhibit carbon steel corrosion by the mechanism of adsorption. The adsorption of sodium silicate and PAMAM obeyed the Langmuir adsorption isotherm. PDP study revealed that addition of the dendrimer and sodium silicate in the corrosive medium affected both anodic as well as cathodic reactions and did not caused any significant change in the value of $E_{\text {corr }}$ suggesting that PAMAM and sodium silicate undertaken in the investigation are mixed type inhibitors. The different combinations of PAMAM and sodium silicate at the concentration of $15 \mathrm{mg} \mathrm{L}^{-1}$ were tested for effect of synergism. Results of the analysis showed that significant enhancement in the inhibition performance of PAMAM dendrimer was observed in the different combination of sodium silicate. The PAMAM exhibited inhibition efficiencies of only $52.2 \%, 55.1 \%$ and $57.5 \%$ at 15,20 and $30 \mathrm{mg} \mathrm{L}^{-1}$ concentrations, respectively. However, the PAMAM and sodium silicate (9:6 ratio) combination shows a substantial synergism. 
The PAMAM and sodium silicate (in 9:6 ratio) composite showed optimum inhibition efficiencies of $91.7 \%, 92.4 \%$ and $97.3 \%$ at the same studied concentrations i.e. at 15, 20 and $30 \mathrm{mg} \mathrm{L}^{-1}$, respectively. The EIS study reveals that PAMAM and sodium silicate act as interface corrosion inhibitors and their adsorption on the metal/ electrolyte interfaces have been supported by EDX and AFM analysis. EDX study suggests that in the presence of PAMAM and sodium silicate composite intensity for the EDX signals correspond to the carbon, nitrogen and oxygen increases on increasing composite concentration. AFM analysis showed that after $72 \mathrm{hrs}$ immersion time metallic surface badly rough and damaged due to free acid attack in the absence of inhibitor. However, in the presence of composite the surface became smoother due to adsorption of composite at metal surface.

El-Mahdy et al. [36] synthesized new water soluble dendritic polyether abbreviated as HPAE-PEG600 using pentaerythritol tetracrylate and diethanolamine as precursor molecule via Michael addition reaction. The synthesized dendrimer was characterized using FT-IR, ${ }^{1} \mathrm{H}$ and ${ }^{13} \mathrm{C}$ NMR techniques. The inhibition characteristics of synthesized HPAE-PEG600 for steel corrosion in $1 \mathrm{M}$ hydrochloric acid was investigated by potentiodynamic polarization and electrochemical impedance spectroscopic methods. The electrochemical impedance spectroscopic study revealed that HPAE-PEG600 adsorbed on the metal-electrolyte interfaces and acts as interface corrosion inhibitors. A substantial increase in the values of charge transfer resistance $\left(R_{\mathrm{ct}}\right)$ was observed in the presence of HPAE-PEG600. The $R_{\mathrm{ct}}$ values increases from $5.9 \mathrm{Ohm}(69.49 \%)$ to $10.4 \mathrm{Ohm}(82.17 \%)$ on increasing HPAE-PEG600 concentration from 50 to $250 \mathrm{ppm}$. During the polarization study it was observed that presence of HPAE-PEG600 in the corrosive test solution causes significant change in the values of anodic and cathodic Tafel slopes without affecting the values of corrosion potential too much. This observation suggest that HPAE-PEG600 is an effective and mixed type inhibitor.

The ethylenediamine cored imidazoline based dendrimeric polymer with four terminal imidazole rings, tetra-imidazoline (DDTI) was synthesized and investigated as good corrosion inhibitor for carbon steel in $\mathrm{CO}_{2}$ medium using weight loss, PDP, EIS and SEM methods [37]. Results manifested that the DDTI acts as good corrosion inhibitor for carbon steel and showed the optimum inhibition efficiency of $98.29 \%$ at $400 \mathrm{mg} \mathrm{L}^{-1}$ concentration. Results further showed that DDTI forms multi-layered film over the metallic surface which isolates the surface from corrosive environment and save from corrosion. The interfacial nature of the DDTI was determined by EIS study in which significant increase in the values of charge transfer resistance $\left(R_{\mathrm{ct}}\right)$ was observed in the presence of the DDTI. The adsorption of the DDTI follows the most common Langmuir adsorption isotherm. The large negative values of standard Gibbs' free energy for adsorption $\left(\Delta G^{0}\right)$ showed that DDTI has strong tendency of spontaneous and chemical adsorption on the metal surface. For DDTI the values of $\Delta G^{0}$ ranges from $-40.0 \mathrm{~kJ} \mathrm{~mol}^{-1}$ to $-45.77 \mathrm{~kJ} \mathrm{~mol}^{-1}$. The morphology of the metal surfaces have also been tested using SEM analysis where the huge smoothness in the surface morphology of the metal surface 
was obtained in the presence of different concentrations of DDTI. This increased surface smoothness revealed that DDTI inhibit metallic corrosion through adsorption mechanism. Polarization study revealed that DDTI affected both the values of anodic as well as cathodic Tafel slopes, however, anodic Tafel slopes values were more affected as compared to the cathodic Tafel slopes values suggesting that DDTI is a predominantly anodic type inhibitor.

In another study [38], our research group has tested the inhibition effect of two ammonia based PAMAM dendrimers namely, DEND- $G_{0}$ and DENG-G $G_{1}$ on mild steel corrosion using weight loss, electrochemical, SEM, EDX, AFM and DFT studies. Results showed that both the tested dendrimers act as efficient inhibitors for mild steel corrosion in hydrochloric acid solution. Inhibition performance of the dendrimers was tested at different concentrations ranging from 10 to $60 \mathrm{ppm}$, however after $50 \mathrm{ppm}$ concentration there was no much change in the inhibition performance which suggested that $50 \mathrm{ppm}\left(\mathrm{mg} \mathrm{L}^{-1}\right)$ was the optimum concentration. The weight loss investigation showed that inhibition efficiencies of the both dendrimers enhances on increasing their concentrations. Among the dendrimers taken under consideration, $D E N G-G_{1}$ exhibited slightly higher inhibition efficiency as compared to DENG-G $\mathrm{G}_{0}$ which is might be resulted due to higher molecular which in turns causes high surface coverage for DENG-G $G_{1}$ as compared to the DEND-G ${ }_{0}$ which have slightly lower molecular size and lesser numbers of heteroatoms. Several adsorption isotherms were tested in order to find best for the both tested dendrimers, among which Langmuir isotherm gave the best fit. The values of $K_{\text {ads }}$ for DENG-G $\mathrm{G}_{0}$ vary from $19.0 \times 10^{3} \mathrm{M}^{-1}$ to $5.34 \times 10^{3} \mathrm{M}^{-1}$, while for $\mathrm{DENG}_{1} \mathrm{G}_{1} K_{\mathrm{ads}}$ values vary from $36.31 \times 10^{3} \mathrm{M}^{-1}$ to $7.13 \times 10^{3} \mathrm{M}^{-1}$ from varying temperature from 308 to $338 \mathrm{~K}$. The high value of $K_{\text {ads }}$ suggested that investigated dendrimers have strong tendency of adsorption. The values of standard Gibbs' free energy was evaluated at different temperature for both the dendrimers using formula $\Delta G_{\mathrm{ads}}^{\mathrm{o}}=-R T \ln \left(55.5 K_{\mathrm{ads}}\right)$. The calculated $\Delta G^{0}$ values vary from -34.28 to $-35.52 \mathrm{~kJ} \mathrm{~mol}^{-1}$ for DENG-G , while for DENG-G $_{1}, \Delta G^{0}$ values vary from -37.18 to -35.24 . The negative sign of the $\Delta G^{\circ}$ for both the dendrimers suggested that they are spontaneously adsorbed over the mild steel surface.

The $\Delta G^{0}$ values for both the dendrimers are more than $-40 \mathrm{~kJ} \mathrm{~mol}^{-1}$ and less than $-20 \mathrm{~kJ} \mathrm{~mol}^{-1}$ suggesting that both the dendrimers adsorbed on the metallic surface by physiochemisorption mechanism. The values of activation energy $\left(E_{\mathrm{a}}\right)$, enthalpy of adsorption $\left(\Delta H^{*}\right)$ and entropy of adsorption $\left(\Delta S^{*}\right)$ were also derived for studied dendrimers by the weight loss experiments at different temperatures ranging from 308 to $338 \mathrm{~K}$. The values of $E_{\mathrm{a}}$ were higher for $\mathrm{DENG}^{-G_{0}}\left(55.47 \mathrm{~kJ} \mathrm{~mol}^{-1}\right)$ and $\mathrm{DENG}_{1}$ $\left(65.42 \mathrm{~kJ} \mathrm{~mol}^{-1}\right)$ as compared to the $E_{\mathrm{a}}$ value of blank $\left(28.48 \mathrm{~kJ} \mathrm{~mol}^{-1}\right)$. The superior values of $E_{\mathrm{a}}$ for inhibited solution indicate that in presence of dendrimers metallic dissolution has become difficult due to formation of energy barrier. Similar interpretation was derived from the increased values of $\Delta H^{*}$ and $\Delta S^{*}$ in the presence of dendrimer as compared to in their absence. 
The investigated dendrimers were also subjected to electrochemical studies using EIS and PDP methods. The EIS and PDP studies carried out only at their optimum concentration i.e. at $50 \mathrm{mg} \mathrm{L}^{-1}$ (ppm) concentration. EIS study revealed that both the dendrimers enhance the value of charge transfer resistance in their presence and acted as interface type of corrosion inhibitors. Presence of the single semicircle in the Nyquist plots of the inhibited and uninhibited mild steel specimens suggests that corrosion of mild steel in the tested solution involves single charge transfer mechanism. The values of $R_{\mathrm{ct}}$ were $173.7 \Omega \mathrm{cm}^{2}$ and $259.2 \Omega \mathrm{cm}^{2}$ for DENG-G ${ }_{0}$ and DENG-G ${ }_{1}$, respectively at $50 \mathrm{mg} \mathrm{L}^{-1}$ concentration. The increased value of $R_{\mathrm{ct}}$ and decreased value of $C_{\mathrm{dl}}$ i.e. double layer capacitance suggested that dendrimers adsorb on the metal-electrolyte interfaces and thereby form the protective film which protect metals from corrosion.

The EIS study was further supported by PDP study, where significant decrease in the corrosion current densities was observed in the presence of dendrimers. Presence of dendrimers at their optimum concentrations affect the anodic and cathodic Tafel curves without significant change in the values of corrosion potential $\left(E_{\text {corr }}\right)$ suggesting that both the dendrimers are behaved as mixed type corrosion inhibitors. Moreover, the shape of polarization curves for inhibited and uninhibited metal specimens are similar which again suggests that both the investigated dendrimers inhibit corrosion by adsorbing on the metal surface without changing the mechanism of metal dissolution. The weight loss, EIS and PDP analyses were supported by scanning electron microscopy (SEM) study. The corroded mild steel specimens after 3 hrs immersion time were analysed under SEM method at 500x magnification. The metal surface corroded in absence of dendrimers showed very high surface roughness due to free acid attack. However, in the presence of dendrimers at $50 \mathrm{mg} \mathrm{L}^{-1}$ concentration the surfaces become smoother which attributed due to formation of the protective film by dendrimers. The finding of SEM analysis was supported by EDX and AFM studies. The authors observed that AFM images of inhibited metal specimens are relatively smooth as compared to the uninhibited metal specimen. Increased intensity for nitrogen and oxygen in the EDX spectra of inhibited mild steel as compared to uninhibited metal specimen showed that dendrimers inhibit corrosion by adsorption mechanism.

The experimental results of weight loss, electrochemical (EIS and PDP) and surface (SEM, AFM and EDX) was supported by DFT based quantum chemical calculations. The DFT analysis were performed on the protonated and neutral forms of the dendrimers. A good agreement between DFT and experimental results were observed. Several DFT based parameters such as energy of frontier molecular orbital ( $E_{\mathrm{HOMO}}$ and $\left.E_{\mathrm{LUMO}}\right)$, energy band gap $(\Delta E)$ global hardness $(\eta)$ and softness $(\sigma)$ and dipole moment $(\mu)$ were derived and discussed in order to correlate the sequence of inhibition efficiency derived from experimental means. Both the tested dendrimers have high value of $E_{\mathrm{HOMO}}$ and low value of $E_{\mathrm{LUMO}}$ which suggested that they have strong adsorption and interactions tendency with the metallic surface. The values of $E_{\mathrm{HOMO}}$ and $E_{\mathrm{LUMO}}$ are accordance to the experimentally determined inhibition efficiency. The DENG-G ${ }_{1}$ has lesser value of $\Delta E$ as compared to the 
DENG- $\mathrm{G}_{0}$ suggesting that DENG-G $\mathrm{G}_{1}$ is more reactive as showed better adsorption tendency as compared to the DENG-G $\mathrm{G}_{0}$.

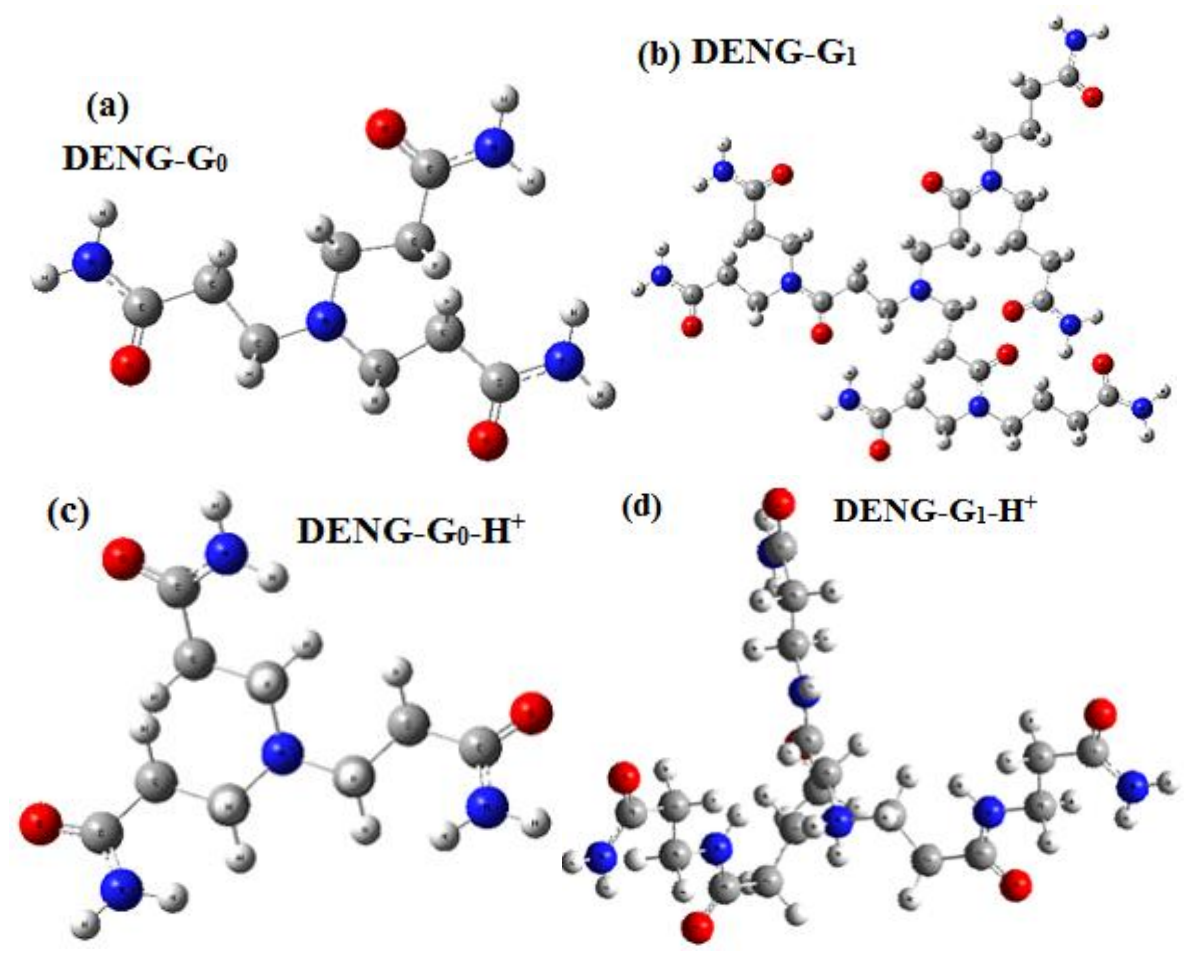

Figure 3. Optimized structure of neutral as well as protonated forms of dendrimers:

(a) DEND-G ${ }_{0}$, (b) DEND-G ${ }_{1}$, (c) DENG-G ${ }_{0}-\mathrm{H}^{+}$and (d) DENG-G ${ }_{1}-\mathrm{H}^{+}$.

The higher dipole moment value for DENG-G1 as compared to the DENG-G0 indicated that DENG-G1 has more tendency of polarizability and will cover larger surface area as compared to the DENG-G0. The global hardness values for DENG-G1 and DENGG0 were 0.130 Hartree and 0.280 Hartree, respectively. These results showed that DENGG1 is chemically more resistant and therefore showed fewer interactions with the mild steel surface as compared to the DENG-G0. Similar conclusion can be derived from the value of global softness $(\sigma)$; DENG-G1 has higher value of global softness and therefore is more reactive as compared to the DENG-G0 with lower value of global softness. The optimized and frontier molecular orbital pictures (HOMO and LUMO) of the tested dendrimers are shown in Figures 3 and 4. From the frontier molecular orbital diagrams it was observed that HOMO and LUMO electrons densities are distributed over the entire part of the dendrimer molecules indicating that almost whole part of the molecules donate to the metal surface as well as accept electrons from the metal surface. 

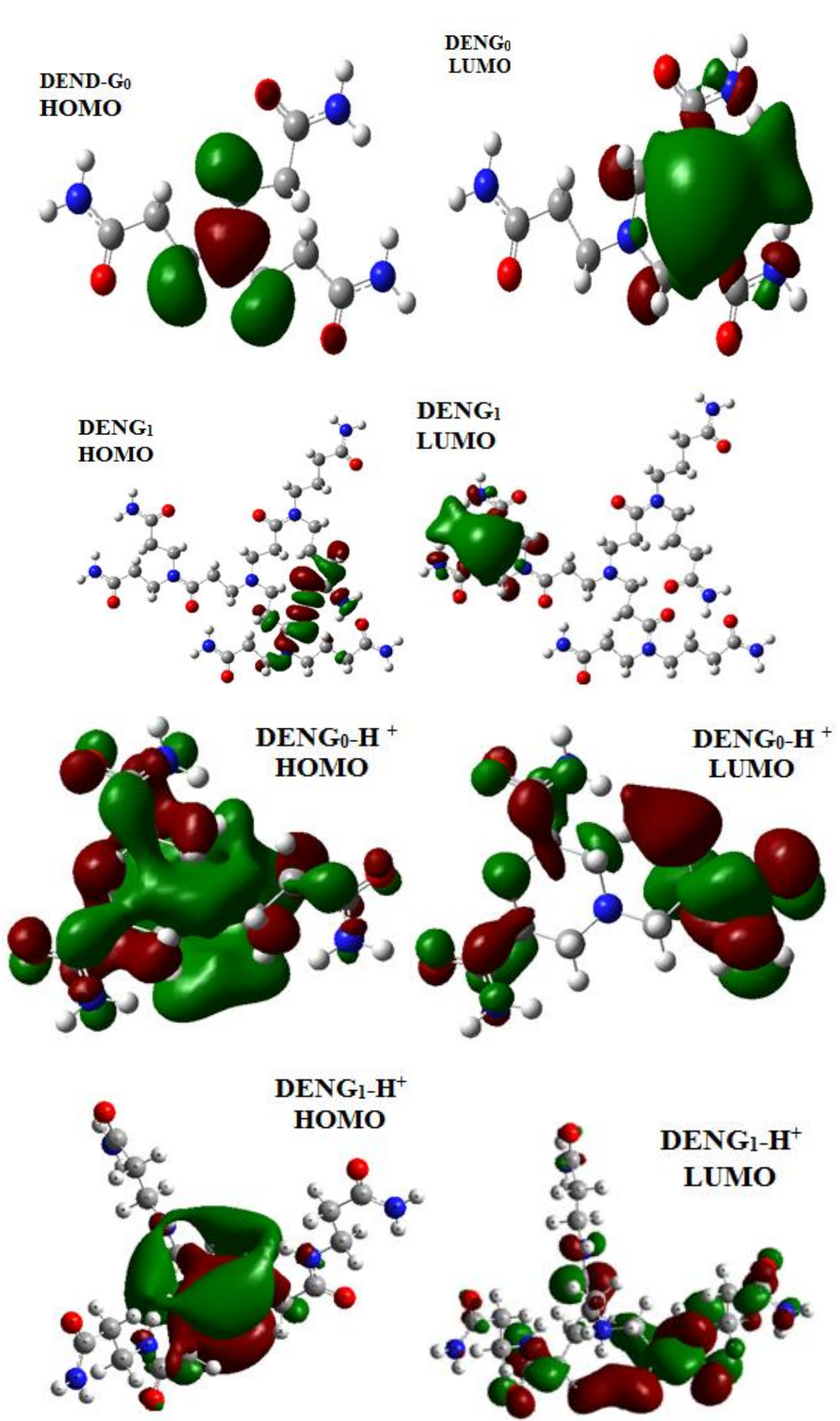

Figure 4. Frontier molecular orbitals of neutral and protonated forms of DEND-G ${ }_{0}$ (a) and DEND-G $_{1}$ (b). 


\section{Dendrimers as scale inhibitors}

Apart from the above use as solution phase corrosion inhibitors, the dendrimers have been extensively used as scale inhibitors particularly for silica. Demadis [39] studied the inhibition properties of a PAMAM dendrimer with generations $0.5,1,1.5,2$ and 2.5 for silica scale growth. Experimental results showed that scale inhibition tendency of the PAMAM dendrimers mainly influence by the nature and number of the peripheral groups. The PAMAM dendrimer becomes effective as low as $40 \mathrm{ppm}$ concentration by forming the silica-PAMAM composite. The loss in the inhibition performance was observed over the time due to co-precipitation and entrapment in the silica matrix. The results showed that presence of the terminal $-\mathrm{NH}_{2}$ groups are responsible for the high scale inhibitory property of the PAMAM dendrimer. The anti-scaling property of the PAMAM dendrimers in combination with carboxymethylinulin (CMI) has also been investigated other group of authors [40]. The inhibitory effect of the PAMAM is again attributed due to formation of PAMAM-silica insoluble composite on the surface. Neofotistou and Demadis [41] studied the anti-scaling behaviour of a PAMAM dendrimer by a combined effect of $-\mathrm{COOH}$ and $-\mathrm{NH}_{2}$ groups.

\section{Conclusion}

From the ongoing discussion it can be concluded that dendrimers are ideal polymeric candidates to replace tradition organic corrosion inhibitors due to their ease synthesis, high solubility in the polar solvents, and presence of polar functional groups as the periphery through which they can easily adsorb on the metallic surfaces. The dendrimers are polymeric materials and therefore associate with very high molecular weight with several adsorption centres and high surface area due to their nano-sized nature. Generally, dendrimers inhibits metallic corrosion by adsorbing and forming the insoluble metaldendrimer film which separates metal from corrosive surrounding. The adsorption of the dendrimers on metallic surface often obey the Langmuir adsorption isotherm. Literature survey reveals that dendrimers mostly acted as interface inhibitors i.e. adsorb at metalelectrolyte interfaces in which their terminal functional groups act as adsorption centres. PAMAM is the most common dendrimer which has been investigated as metal corrosion inhibitors. Most of the investigated dendrimers acted as mixed type corrosion inhibitors. The inhibition efficiency of the dendrimers have been investigated using several experimental such as weight loss, EIS, PDP, AFM, SEM and EDX as well as computational such as MD and DFT methods. The anti-scaling behaviour of few PAMAM dendrimers have also been studied particularly for silica. Literature results showed that PAMAM dendrimers become effective anti-scalants by forming insoluble silica-PAMAM dendrimers. 


\section{Acknowledgements}

Chandrabhan Verma, gratefully acknowledges the North-West University (Mafikeng Campus) South Africa for providing financial supports for the study under Postdoctoral Fellowship scheme.

\section{References}

1. R.W. Revie, Corrosion and corrosion control: an introduction to Corros. Sci. and engineering, John Wiley \& Sons, 2008.

2. Y.M. Panchenko and A. Marshakov, Long-term prediction of metal corrosion losses in atmosphere using a power-linear function, Corros. Sci., 2016, 109, 217-229.

3. C. Verma, E.E. Ebenso and M. Quraishi, Ionic liquids as green and sustainable corrosion inhibitors for metals and alloys: an overview, J. Mol. Liq., 2017, 233, $403-$ 414.

4. C. Verma, L. Olasunkanmi, E.E. Ebenso and M. Quraishi, Substituents effect on corrosion inhibition performance of organic compounds in aggressive ionic solutions: A review, J. Mol. Liq., 2018, 251, 100-118.

5. A. Bakkar and S. Ataya, Corrosion behaviour of stainless steel fibre-reinforced copper metal matrix composite with reference to electrochemical response of its constituents, Corros. Sci., 2014, 85, 343-351.

6. I. Obot, D. Macdonald and Z. Gasem, Density functional theory (DFT) as a powerful tool for designing new organic corrosion inhibitors. Part 1: an overview, Corros. Sci., 2015, 99, 1-30.

7. F. Ansari, C. Verma, Y. Siddiqui, E. Ebenso and M. Quraishi, Volatile corrosion inhibitors for ferrous and non-ferrous metals and alloys: A review, Int. J. Corros. Scale Inhib., 2018, 7, 126-150. doi: 10.17675/2305-6894-2018-7-2-2

8. C. Verma, L.O. Olasunkanmi, E.E. Ebenso, M.A. Quraishi and I.B. Obot, Adsorption behavior of glucosamine-based, pyrimidine-fused heterocycles as green corrosion inhibitors for mild steel: experimental and theoretical studies, J. Phys. Chem. C, 2016, 120, 11598-11611.

9. L.O. Olasunkanmi, I.B. Obot, M.M. Kabanda and E.E. Ebenso, Some quinoxalin-6-yl derivatives as corrosion inhibitors for mild steel in hydrochloric acid: experimental and theoretical studies, J. Phys. Chem. C, 2015, 119, 16004-16019.

10. A. Brenna, M. Ormellese, M. Pedeferri and F. Bolzoni, Effect of binary mixtures on chloride induced corrosion of rebars in concrete, Int. J. Corros. Scale Inhib., 2018, 7, 151-164. doi: $10.17675 / 2305-6894-2018-7-2-3$

11. V. Golovin, A. Ilyin, A. Aliev, V. Shchelkov and V. Rabinkov, A study on the desorption of phosphonic acids, corrosion inhibitor precursors, from polymer microcapsules, Int. J. Corros. Scale Inhib., 2018, 7, 165-174. doi: 10.17675/2305$\underline{6894-2018-7-2-4}$ 
12. B. Mazères, C. Desgranges, C. Toffolon-Masclet and D. Monceau, Experimental study and numerical simulation of high temperature $\left(1100-1250^{\circ} \mathrm{C}\right)$ oxidation of prioroxidized zirconium alloy, Corros. Sci., 2016, 103, 10-19.

13. X. Yu, B. Jiang, H. Yang, Q. Yang, X. Xia and F. Pan, High temperature oxidation behavior of Mg-Y-Sn, Mg-Y, Mg-Sn alloys and its effect on corrosion property, Appl. Surf. Sci., 2015, 353, 1013-1022.

14. Z. Tao, S. Zhang, W. Li and B. Hou, Corrosion inhibition of mild steel in acidic solution by some oxo-triazole derivatives, Corros. Sci., 2009, 51, 2588-2595.

15. K. Rasheeda, D. Vijaya, P. Krishnaprasad and S. Samshuddin, Pyrimidine derivatives as potential corrosion inhibitors for steel in acid medium - An overview, Int. J. Corros. Scale Inhib., 2018, 7, 48-61. doi: 10.17675/2305-6894-2018-7-1-5

16. M. Finšgar and J. Jackson, Application of corrosion inhibitors for steels in acidic media for the oil and gas industry: a review, Corros. Sci., 2014, 86, 17-41.

17. S.H. Zaferani and M.R. Shishesaz, Corrosion Inhibition of Carbon Steel in Acidic Solution by Alizarin Yellow GG “AYGG”, J. Pet. Environ. Biotechnol., 2014, 5, 1-5.

18. H. Park, K.Y. Kim and W. Choi, Photoelectrochemical approach for metal corrosion prevention using a semiconductor photoanode, J. Phys. Chem. B, 2002, 106, 47754781.

19. M. Ihara, H. Nishihara and K. Aramaki, The inhibition of iron corrosion in acid solutions using bismuth (III) chloride, Corros. Sci., 1992, 33, 1267-1279.

20. O.K. Abiola, N. Oforka, E. Ebenso and N. Nwinuka, Eco-friendly corrosion inhibitors: The inhibitive action of Delonix Regia extract for the corrosion of aluminium in acidic media, Anti-Corros. Meth. Mater., 2007, 54, 219-224.

21. F. Cotting and I.V. Aoki, Smart protection provided by epoxy clear coating doped with polystyrene microcapsules containing silanol and Ce (III) ions as corrosion inhibitors, Surf. Coat. Technol., 2016, 303, 310-318.

22. F.S. de Souza and A. Spinelli, Caffeic acid as a green corrosion inhibitor for mild steel, Corros. Sci., 2009, 51, 642-649.

23. C. Verma, L.O. Olasunkanmi, T.W. Quadri, E.-S.M. Sherif and E.E. Ebenso, Gravimetric, Electrochemical, Surface Morphology, DFT, and Monte Carlo Simulation Studies on Three N-Substituted 2-Aminopyridine Derivatives as Corrosion Inhibitors of Mild Steel in Acidic Medium, J. Phys. Chem. C, 2018, 122, 11870-11882.

24. O.A. Goncharova, A.Yu. Luchkin, Yu.I. Kuznetsov, N.N. Andreev, N.P. Andreeva and S.S. Vesely, Octadecylamine, 1,2,3-benzotriazole and a mixture thereof as chamber inhibitors of steel corrosion, Int. J. Corros. Scale Inhib., 2018, 7, 203-212. doi: 10.17675/2305-6894-2018-7-2-7

25. Y. Xu, S. Zhang, L. Guo, B. Tan, C. Liao, Y. Zhou and L. Madkour, Halogen-substituted pyrazolo-pyrimidine derivatives as corrosion inhibitors for copper in sulfuric acid solution, Int. J. Corros. Scale Inhib., 2018, 7, 236-249. doi: 10.17675/2305-6894-20187-2-9 
26. Yu.I. Kuznetsov, Organic corrosion inhibitors: where are we now? A review. Part IV. Passivation and the role of monoand diphosphonates, Int. J. Corros. Scale Inhib., 2017, 6, 384-427. doi: 10.17675/2305-6894-2017-6-4-3

27. M. El Azzouzi, A. Aouniti, M. El Massaoudi, S. Radi, B. Hammouti, H. Bendaif and Y. El Ouadi, Inhibition effect of 1,1'-(pyridine-2,6-dihylbis(methylene))bis(5-methyl1-H-pyrazole-3-carboxylic acid) on the corrosion of mild steel in $1 \mathrm{M} \mathrm{HCl}$. Part A: Experimental study, Int. J. Corros. Scale Inhib., 2017, 6, 463-475. doi: 10.17675/23056894-2017-6-4-6

28. N. Wang, W. Ma, Z. Ren, Y. Du, P. Xu and X. Han, Prussian blue analogues derived porous nitrogen-doped carbon microspheres as high-performance metal-free peroxymonosulfate activators for non-radical-dominated degradation of organic pollutants, J. Mater. Chem. A, 2018, 6, 884-895.

29. U. Boas and P.M. Heegaard, Dendrimers in drug research, Chem. Soc. Rev., 2004, 33, 43-63.

30. D. Boris and M. Rubinstein, A self-consistent mean field model of a starburst dendrimer: dense core vs dense shell, Macromolecules, 1996, 29, 7251-7260.

31. T.F. Vandamme and L. Brobeck, Poly(amidoamine) dendrimers as ophthalmic vehicles for ocular delivery of pilocarpine nitrate and tropicamide, J. Controlled Release, 2005, 102, 23-38.

32. A.-M. Caminade, R. Laurent and J.-P. Majoral, Characterization of dendrimers, $A d v$. Drug Delivery Rev., 2005, 57, 2130-2146.

33. K. Khaled, A. Atta and N. Abdel-Shafi, A structure/function study of polyamidoamine dendrimer as a steel corrosion inhibitor, J. Mater. Environ. Sci., 2014, 5, 831-840.

34. C. Verma and M.A. Quraishi, Thermodynamic, Electrochemical and Surface Studies of Dendrimers as Effective Corrosion Inhibitors for Mild Steel in $1 \mathrm{M} \mathrm{HCl}$, Anal. Bioanal. Electrochem., 2016, 8, 104-123.

35. B. Zhang, C. He, X. Chen, Z. Tian and F. Li, The synergistic effect of polyamidoamine dendrimers and sodium silicate on the corrosion of carbon steel in soft water, Corros. Sci., 2015, 90, 585-596.

36. G.A. El-Mahdy, A.M. Atta, H.A. Al-lohedan and A.O. Ezzat, Synthesis of Water Soluble Hyperbranched Poly (amine-ester) as Corrosion Inhibitors for Steel, Int. J. Electrochem. Sci., 2014, 9, 7925-7934.

37. Y. He, Y. Zhou, R. Yang, L. Ma and Z. Chen, Imidazoline derivative with four imidazole reaction centers as an efficient corrosion inhibitor for anti-CO2 corrosion, Russ. J. Appl. Chem., 2015, 88, 1192-1200.

38. C. Verma, E.E. Ebenso, Y. Vishal and M. Quraishi, Dendrimers: A new class of corrosion inhibitors for mild steel in $1 \mathrm{M} \mathrm{HCl}$ : Experimental and quantum chemical studies, J. Mol. Liq., 2016, 224, 1282-1293.

39. K.D. Demadis, A structure/function study of polyaminoamide dendrimers as silica scale growth inhibitors, J. Chem. Technol. Biotechnol., International Research in Process, Environmental \& Clean Technology, 2005, 80, 630-640. 
40. E. Mavredaki, A. Stathoulopoulou, E. Neofotistou and K.D. Demadis, Environmentally benign chemical additives in the treatment and chemical cleaning of process water systems: Implications for green chemical technology, Desalination, 2007, 210, 257265.

41. E. Neofotistou and K.D. Demadis, Use of antiscalants for mitigation of silica (SiO2) fouling and deposition: fundamentals and applications in desalination systems, Desalination, 2004, 167, 257-272. 